\title{
Erfahrungsbericht AOTD-Reisestipendium 2018 am Missouri Orthopaedic Institute, MO, USA
}

\author{
Anna J. Schreiner
}

Die AOTrauma Deutschland vergibt jährlich mindestens ein Reisestipendium zur Förderung des Erfahrungsaustausches und der Weiterbildung bei angesehenen Experten in unserer Disziplin. Im folgenden Artikel berichtet Frau Dr. Anna J. Schreiner, Tübingen von ihren Erfahrungen.

Die Unterstützung durch die AOTrauma Deutschland ermöglichte mir einen 4-wöchigen Aufenthalt am University Hospital (UH) der University of Missouri (Level-I-Trauma Center) sowie dem Missouri Orthopedic Institute (MOI) in Columbia, Missouri im Oktober/November 2018. Die Klinik bildet das komplette Spektrum der Orthopädie und Unfallchirurgie ab und ist der Maximalversorger in Zentral-Missouri mit dem größten freistehenden orthopädischen Zentrum. Das Thompson Laboratory for Regenerative Orthopaedics unter der Leitung von Prof. Jim Cook mit Wissenschaft auf höchstem Niveau ist Teil der Einrichtung.

Geleitet wird das MOI bzw. das Department of Orthopaedic Surgery von Prof. James „jim“ Stannard, seines Zeichens Präsident der AO North America sowie der Editor in Chief von „The Journal of Knee Surgery“. Es war eine Ehre, von dieser lebenden Legende am OP-Tisch lernen zu dürfen. Menschlich wie fachlich sehr beeindruckend waren auch die Zusammentreffen mit Prof. Mauricio Kfuri, das Gesicht der AOTrauma South America, der seine Habilitation als ehem. Humboldt-Stipendiat an der $\mathrm{MHH}$ seinerzeit auf Deutsch verfasst hatte. Des Weiteren hatte ich neben weiteren hervorragenden Chirurgen wie Dr. Crist und Dr. Schweser auch die Gelegenheit, von Prof. Gregory Della Rocca (Chair of the AO North America Research Committee) zu lernen und Fragen zu stellen.

Die Organisation des Aufenthaltes im Vorfeld sowie vor Ort war professionell und unkompliziert. Jeder Fellow oder Observer erhielt einen Wochenplan mit Zuteilung ans UH oder MOI sowie an einen der o.g. Attendings samt Residents bzw. Fellows.

Der Tag begann um 7 Uhr im OP und jeden Mittwoch um 06:30 Uhr mit einer wöchentlichen Fortbildung entweder aus den eigenen Reihen oder mit Guest Speakern. Für Observer in den USA gilt eine strikte No-Touch-Regel. Da Prof. Stannard jedoch eine Sondergenehmigung eingeholt hatte, durften sich Observer am UH/MOI auch mit- einwaschen, was einen näher ans Geschehen brachte. Das reine Observing wiederum ermöglichte eine gelungene Fotodokumentation der spannenden Fälle. Ein Attending operierte pro Tag jeweils alternierend in 2 OP-Sälen und wurde entsprechend durch 2-4 Residents und seine Nurse unterstützt. In der Regel begannen die Residents mindestens mit dem Zugang oder durften sogar unter Aufsicht des Attendings die Operation komplett durchführen, abhängig von der Komplexität der Fälle. Zeitdruck spielte hierbei nie eine Rolle, sodass eine intensive chirurgische Ausbildung in einer wirklich sehr angenehmen Atmosphäre/Stimmung im OP gelebt wurde.

Die personelle und materielle Ausstattung im OP war sehr gut: Jeder Saal war z. B. mit mehreren hochwertigen Bildschirmen ausgestattet, Industrievertreter waren bei jeder OP, die deren Material in Anspruch nahm, mit anwesend und durch andersfarbige Scrubs erkennbar. Die

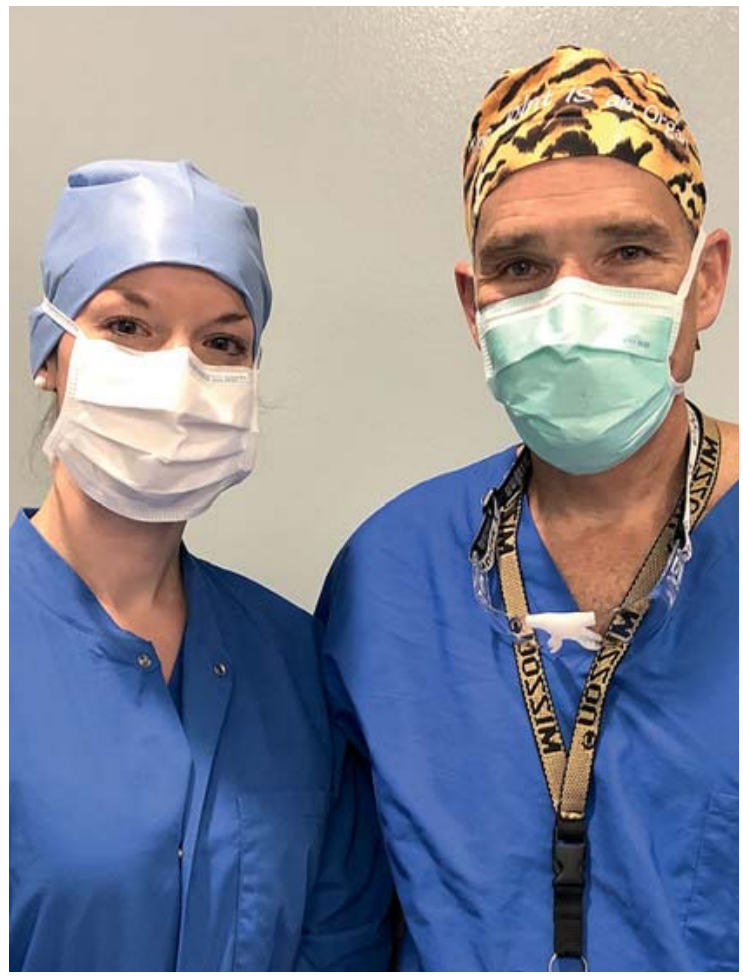

- Abb. 1 Anna Schreiner mit Prof. Stannard. Quelle: Dr. Anna Schreiner 


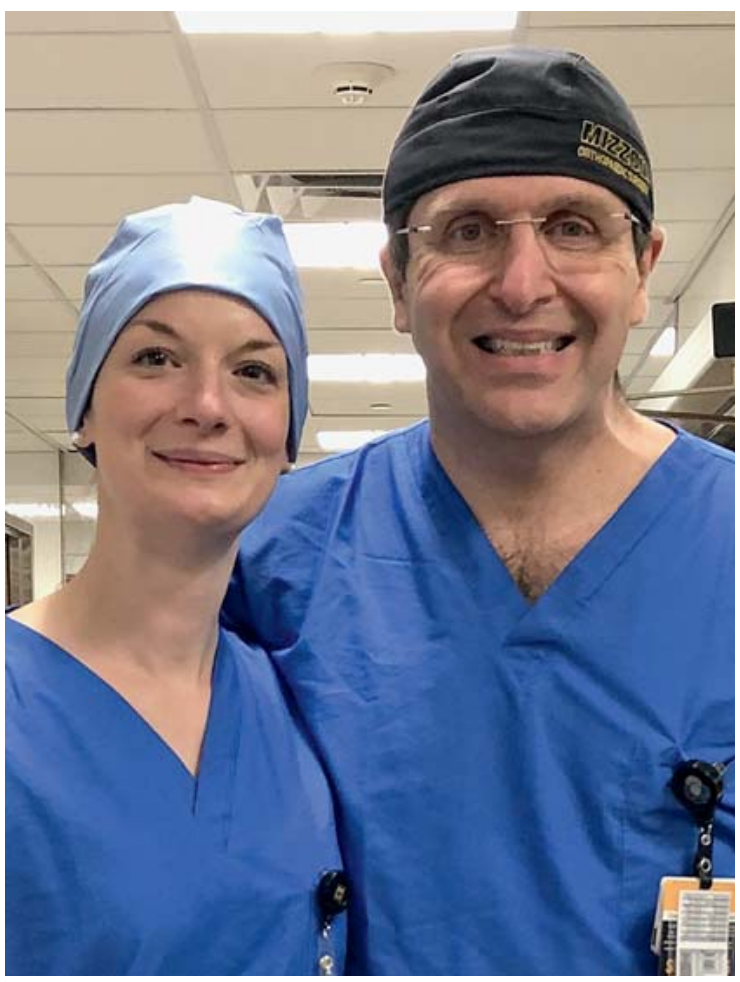

- Abb.2 Anna Schreiner mit Prof. Kfuri. Quelle: Dr. Anna Schreiner

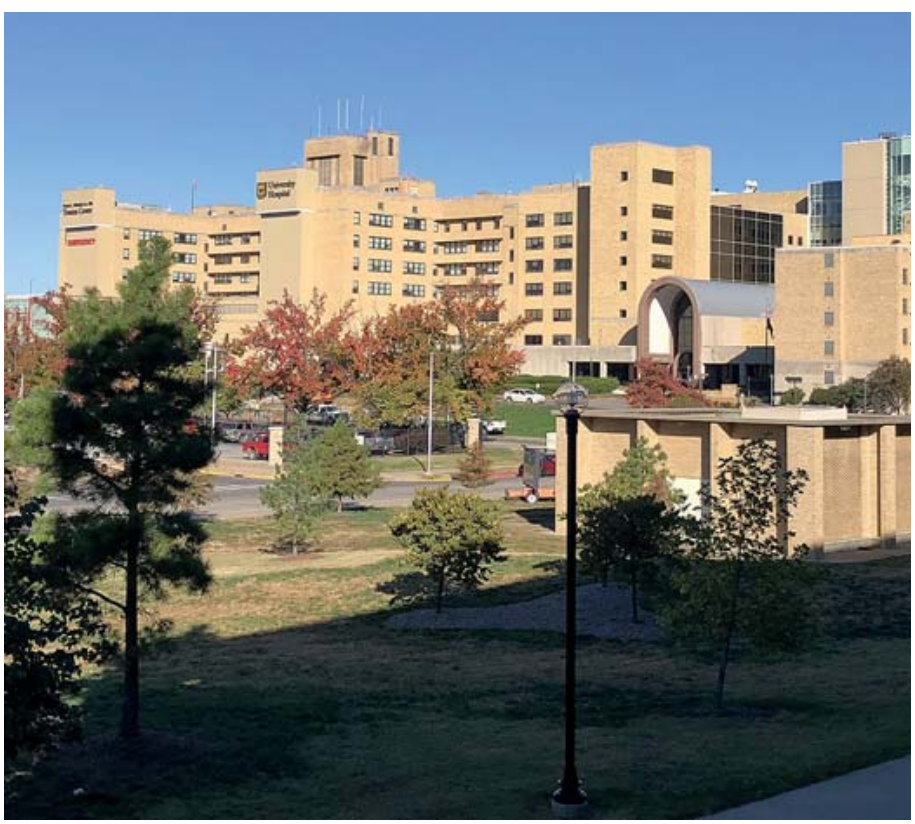

Abb. 4 University Hospital, Columbia, MO. Quelle: Dr. Anna Schreiner

intraoperative Röntgenbildgebung erfolgte stets durch ein eigen hierfür vorgesehenes radiologisches Fachpersonal. Ich war primär im OP eingeteilt, jedoch auch einmal mit Prof. Kfuri in der Sprechstunde. Jeder Attending sieht hier aufgrund der effizienten Organisation zwischen 30-60 Patienten pro Sprechstundentag.

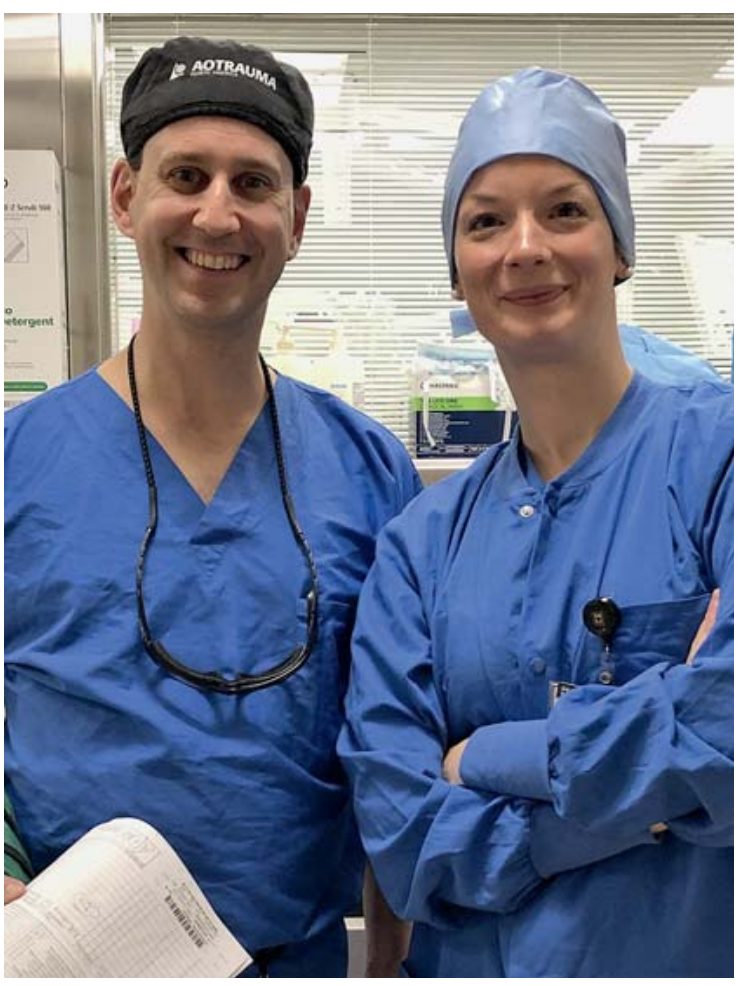

- Abb. 3 Anna Schreiner mit Prof. Della Rocca. Quelle: Dr. Anna Schreiner

Fachlich wie menschlich durfte ich eine hervorragende Teamarbeit miterleben vor dem Hintergrund einer „Culture of Yes“ bzw. Positivität, die sich in vielen Facetten des chirurgischen Alltages widerspiegelte, gepaart mit der grundsätzlich offenen, hilfsbereiten und freundlichen Art der Amerikaner. Die Aufnahme in das Team war sehr herzlich und die Kollegen waren sowohl daran interessiert, ihre Strategien zu demonstrieren, als auch mehr über das deutsche Vorgehen zu erfahren. Auch Thanksgiving durfte ich neben anderen Veranstaltungen und Team-Events mit den dortigen Kollegen und Freunden erleben.

Neben vielen Parallelen zu meiner eigenen Klinik gab es aber auch viel Neues zu lernen bzw. andere Herangehensweisen oder Zugangsoptionen zu erleben. Spannend zu sehen waren z. B. der von Dr. Kfuri entwickelte Extended anterolateral Approach zum Tibiakopf mit einer Osteotomie des lateralen Femurkondylus sowie der von ihm standardmäßig gewählte suprapatellare Zugang im Rahmen der Tibiaschaftmarknagelung. An dieser Stelle ist auch zu erwähnen, dass er zusammen mit Prof. Schatzker dessen Klassifikation für Tibiakopffrakturen aktualisiert und erst neulich publiziert hat. Prof. Kfuri lebt alle AOPrinzipien und teilt als vorbildlicher Lehrer sowohl sein chirurgisches als auch wissenschaftliches Know-how mit allen Kollegen. Prof. Della Rocca führte erstmals eine Radiocapitellar Achilles Disc Arthroplasty im Rahmen einer Radiusköpfchenresektion gemäß den aktuellen Erkennt- 
nissen von Ramazanian et al. durch und demonstrierte neben den klassischen Zugängen am Becken - mehrfach die Option der Kriechschraubenosteosynthese des vorderen Beckenrings gemäß den Veröffentlichungen von Routt et al.

Das besondere Highlight meines Aufenthaltes waren die sog. Bio Joint Replacements. Das „Mizzou Bio Joint Center" ist das einzige Zentrum in den USA, das sog. Gelenktransplantationen in diesem Umfang sowie mit den entsprechenden Resultaten durchführt und ein breites Alters- wie auch Patientenspektrum bis hin zu Profisportlern behandelt. Nach Jahren der grundlagenwissenschaftlichen wie auch biomechanischen Forschung sowie der Entwicklung einer eigenen patentierten Lösung zur GraftKonservierung (das sog. MOPS = Missouri Osteochondral Preservation System) und des Einsatzes von sog. BMAC (bone marrow aspiration concentrate) sind die Kollegen um Prof. Stannard und Prof. Cook in der Lage, schwerpunktmäßig Kniegelenke mono- bis trikompartimentell mittels Leichengrafts erfolgreich zu ersetzen im Sinne eines Large Osteochondral Allograft bei multipel voroperierten Kniegelenken mit entsprechenden Defektsituationen. Ca. 200 Kniegelenke wurden bereits auf diese Art versorgt und sowohl Hüft- als auch Schulter- und Sprunggelenke werden mittlerweile ebenfalls adressiert. Im nächsten Schritt ist u. a. auch die Transplantation mitsamt des vorderen Kreuzbandes angedacht. Im Unterschied zu Deutschland existiert in den USA eine große Graftbank, auf die zugegriffen werden kann. „The joint is an organ“ pflegten Prof. Stannard und Prof. Cook stets zu sagen.

Das Reisestipendium der AOTrauma Deutschland ermöglichte mir die einmalige Gelegenheit, 4 Wochen lang von herausragenden Unfallchirurgen in einem hervorragenden Setting in einem anderen Land lernen zu dürfen und spannende Einblicke in kollegialen und freundschaftlichen Gesprächen zu gewinnen. Neben neuen wissenschaftlichen Erkenntnissen wie auch chirurgischen Impressionen, des Erlebens der universell zum Einsatz kommenden Osteosynthesetechniken der AO sowie einer menschlich-kulturellen Horizonterweiterung hat sich im Rahmen dieses Aufenthaltes sogar die Chance einer Rückkehr im Rahmen eines wissenschaftlichen Stipendiums des MOI ergeben sowie die Möglichkeiten gemeinsamer Studienkooperationen.

\section{Korrespondenzadresse}

\section{Dr. Anna J. Schreiner}

BG Unfallklinik Tübingen

Eberhard Karls Universität Tübingen

aschreiner@bgu-tuebingen.de

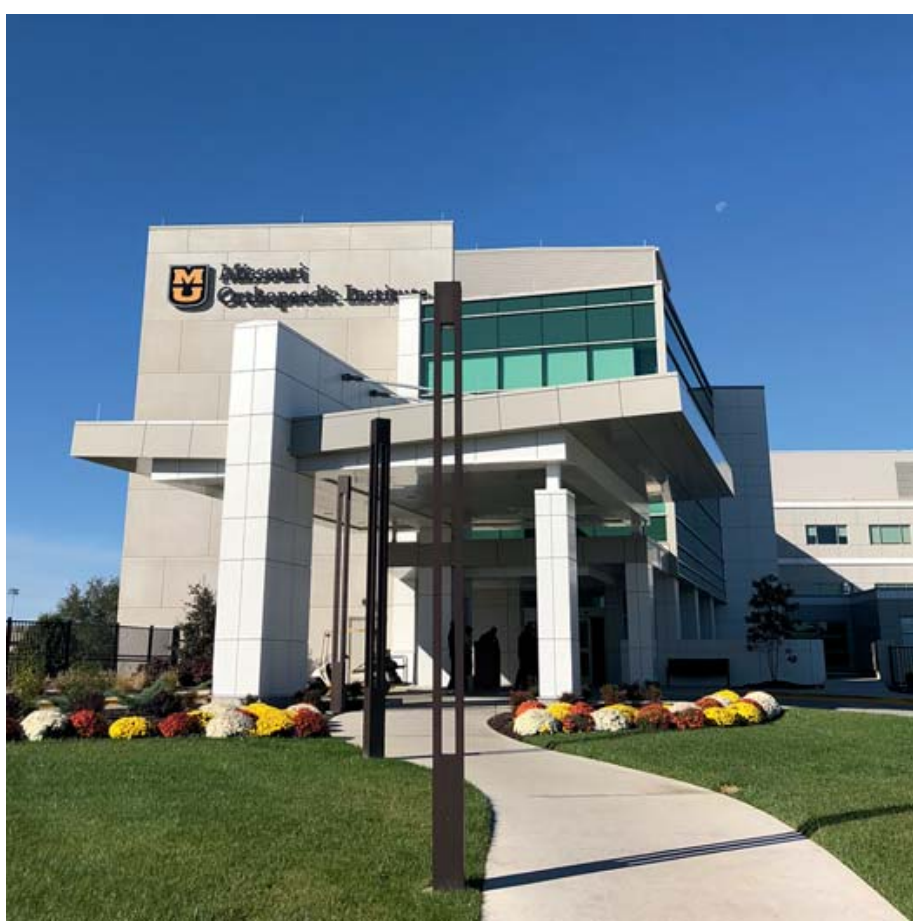

- Abb. 5 Missouri Orthopaedic Institute, Columbia, Missouri. Quelle: Dr. Anna Schreiner

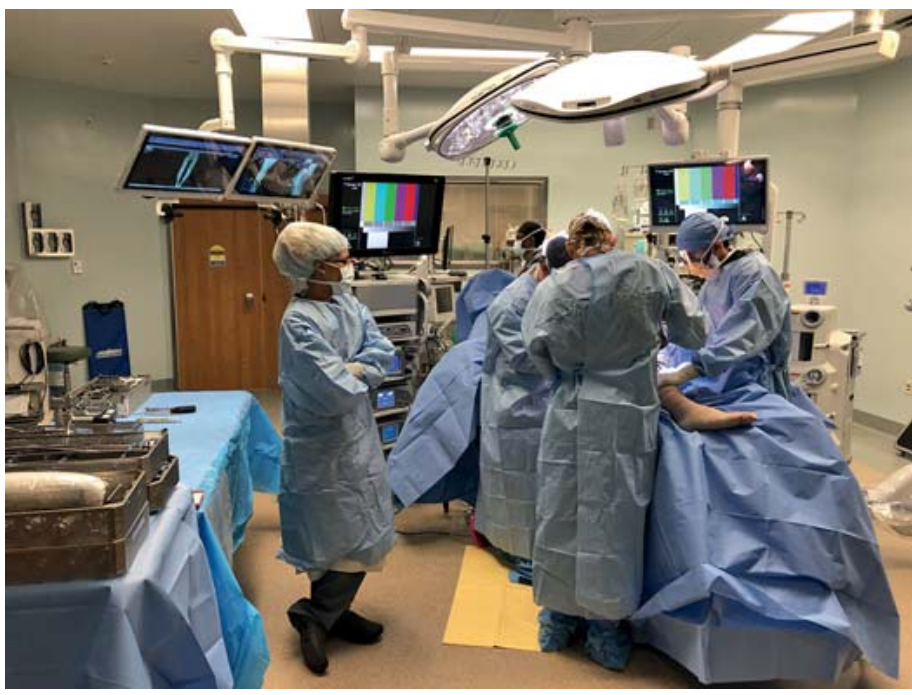

- Abb. 6 Im OP (UH). Quelle: Dr. Anna Schreiner

\section{Bibliografie}

DOI https://doi.org/10.1055/a-0874-4345

OP-JOURNAL 2019; 35: 77-79 @ Georg Thieme Verlag KG Stuttgart · New York ISSN 0178-1715 